\title{
The asymptotic evolution of the stellar merger V1309 Sco: a Blue Straggler in the making?^
}

\author{
Thiago Ferreira ${ }^{1} \dagger$, Roberto K. Saito ${ }^{1}$, Dante Minniti ${ }^{2,3,4}$, María Gabriela Navarro ${ }^{2,5,3}$, \\ Rodrigo Contreras Ramos ${ }^{6,3}$, Leigh Smith ${ }^{7,8}$, Philip W. Lucas ${ }^{7}$ \\ ${ }^{1}$ Departamento de Física, Universidade Federal de Santa Catarina, 88.040-908, Florianópolis, Brazil \\ ${ }^{2}$ Departamento de Ciencias Físicas, Facultad de Ciencias Exactas, Universidad Andres Bello, Av. Fernandez Concha 700, Las Condes, \\ Santiago, Chile \\ ${ }^{3}$ Millennium Institute of Astrophysics, Av. Vicuna Mackenna 4860, 782-0436, Santiago, Chile \\ 4 Vatican Observatory, V00120 Vatican City State, Italy \\ 5 Dipartimento di Fisica, Università degli Studi di Roma "La Sapienza", P.le Aldo Moro, 2, I00185 Rome, Italy \\ 6 Instituto de Astrofisica, Pontificia Universidad Catolica de Chile, Vicuna Mackenna 4860, Macul, Santiago, Chile \\ 7 Institute of Astronomy, University of Cambridge, Madingley Road, Cambridge, CB3 OHA, UK \\ 8 Centre for Astrophysics Research, School of Physics, Astronomy and Mathematics, University of Hertfordshire, College Lane, \\ Hatfield AL10 9AB, UK
}

Accepted XXX. Received YYY; in original form ZZZ

\begin{abstract}
Stellar mergers are estimated to be common events in the Galaxy. The best studied stellar merger case to date is V1309 Sco (= Nova Scorpii 2008) which was originally misclassified as a Nova event. Later identified as the merger of the components of a cool overcontact binary system with $1.52 \mathrm{M}_{\odot}$ and $0.16 \mathrm{M}_{\odot}, \mathrm{V} 1309$ Sco showed an initial period of $P=1.4$ days before the merger. Post-outburst evolution demonstrated that V1309 Sco was unlike the typical Classical Novae and Symbiotic Recurrent Novae with significant dust production around it, and indicated that the system may become a post-AGB (or pre-PN) soon. Here we present a study of V1309 Sco about ten years after the outburst, based on near-IR variability and colour data from the ESO surveys VISTA Variables in the Vía Láctea (VVV) and VVV eXtended (VVVX). We find that reasonable equilibrium in this stellar merger is being reached and that the star has settled into a nearly constant magnitude. A dramatic change in its near-IR colours from $\left(J-K_{\mathrm{S}}\right)=1.40$ in 2010 to $\left(J-K_{\mathrm{S}}\right)=0.42$ in 2015 and a possible low amplitude periodic signal with $P=0.49$ days in the post-outburst data are consistent with a "blue straggler" star, predicted to be formed from a stellar merger.
\end{abstract}

Key words: ephemerides — infrared: stars — surveys — (stars:) novae, cataclysmic variables - techniques: photometric

\section{INTRODUCTION}

Stellar mergers have been estimated to be luminous and common events, however, those phenomena have somehow not been efficiently probed by previous Galactic surveys (e.g. Kochanek et al. 2014). Due to a great variety of binary systems configurations (e.g. Paczyński 1971), it is equally expected a wide variety of possible stellar merges scenarios.

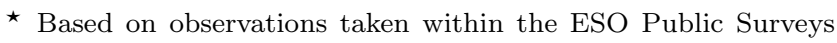
VVV and VVVX, Programme IDs 179.B-2002 and 198.B-2004, respectively.

$\dagger$ E-mail: t.ferreira@astro.ufsc.br
Furthermore, the detailed study of specific cases may lead to the discovery of even new classification of not previously identified objects as such Pietrukowicz et al. (2017).

V1309 Sco, also known as Nova Scorpii 2008 ${ }^{1}$, is the best studied stellar merger case to date, which has been originally discovered as a Nova event by Nakano et al. (2008) and later identified as the merger of the components of a cool overcontact binary system by Tylenda et al. (2011), where their critical Roche lobe is filled out and they share a common envelope. Making use of the OGLE photometry

\footnotetext{
${ }^{1}$ http://simbad.u-strasbg.fr/simbad/sim-id?Ident=v1309+sco\&
} 
(see Udalski et al. 2008), they showed that the progenitor of V1309 Sco was an eclipsing contact binary system with an initial period of $P=1.4$ days. Afterwards, Nandez et al. (2014) demonstrated the high mass ratio of the system, with components of $1.52 \mathrm{M}_{\odot}$ and $0.16 \mathrm{M}_{\odot}$.

Analysing the post-outburst absorption and emission lines of V1309 Sco's spectra evolution, Mason et al. (2010) demonstrated that the system was unlike the typical Classical Novae and Symbiotic Recurrent Novae. ALMA observations at the V1309 Sco region indicated that the system may become a post-AGB (or pre-PN) soon, moreover, yield a kinematic distance of $2.1 \mathrm{kpc}$ for this object (Kamiński et al. 2018). Besides this, there is a significant dust production around V1309 Sco, originated by the merger of the overcontact binary progenitor, and the later evolution of the merger was found to be peculiar (Nichols et al. 2012; McCollum et al. 2014; Tylenda et al. 2016; Zhu et al. 2016). As the evolution of stellar merger systems is not well known yet, V1309 Sco has become an ideal specific example to conduct a detailed study about ten years after the outburst.

The near-IR light curves of the ESO survey VISTA Variables in the Vía Láctea (VVV Survey; Minniti et al. 2010; Saito et al. 2012), and of its complementary survey VVV eXtended (VVVX Survey, Minniti 2018), reach typical magnitude $K_{\mathrm{S}}=17-18 \mathrm{mag}$, which offers a new way to probe luminous stellar merger throughout the Milky Way's bulge and its adjacent disk. Indeed we are starting to unveil interesting high amplitude variable objects, such as the VVV-WIT-06 (Minniti et al. 2017; Banerjee et al. 2018), that has been proposed to be either a supernova, red novae or a merger event. Here we present a study of V1309 Sco about ten years after the outburst, based on near-IR variability and colour data from the VVV Survey.

\section{THE VVV NEAR-IR OBSERVATIONS}

V1309 Sco is located in the Galactic Bulge, at coordinates RA, DEC (J2000): 269.38724, -30.71945 deg., corresponding to Galactic coordinates $l, b: 359.7854^{\circ},-3.1346^{\circ}$, within the VVV tile b291. The V1309 Sco's field was observed by VVV in five near-IR filters in $2010\left(J H K_{\mathrm{S}}\right)$ and $2015\left(Z Y J K_{\mathrm{S}}\right)$ plus a variability campaign in $K_{\mathrm{S}}$-band carried out with a total of 163 epochs spanning from July 082010 to March 28 2017, where 2016 and 2017 observations were provided by the VVVX Survey. The extinction towards the region of V1309 Sco is $\mathrm{A}_{K s}=0.29 \pm 0.11 \mathrm{mag}$ according to VVV extinction maps (Gonzalez et al. 2012), corresponding to $\mathrm{A}_{V}=2.46$ in the optical according to Cardelli et al. (1989) extinction law. Other estimates for the extinction on the target's position are $\mathrm{A}_{K}=0.37 \mathrm{mag}$ according to Schlafly \& Finkbeiner (2011) and $\mathrm{A}_{K}=0.45 \mathrm{mag}$ from Schlegel et al. (1998). The VVV near-IR colour image and the $\mathrm{K}_{\mathrm{S}}$ frame for a $\sim 1.5^{\prime} \times 1.0^{\prime}$ area centred on V1309 Sco are shown in Figure 1.

Due to high crowding at the position of V1309 Sco, the standard VVV aperture photometry provided by the Cambridge Astronomical Survey Unit (CASU; GonzálezFernández et al. 2018) doesn't work properly, especially because of the presence of a nearby source of similar magnitude, which seems to blend with our target depending on the sky seeing. Therefore, PSF photometry on the VVV images

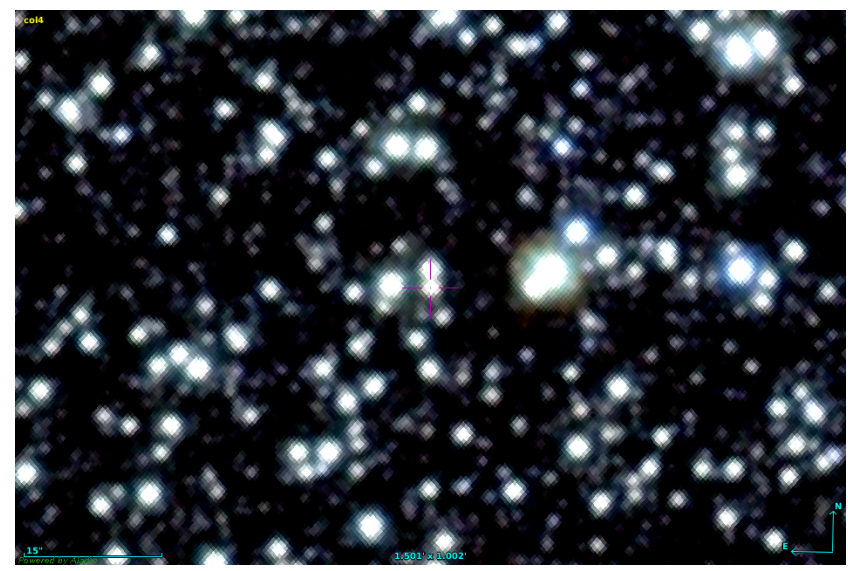

Figure 1. VVV JHKs near-IR false colour image of the V1309 Sco's. The FoV is $\sim 1.5^{\prime} \times 1.0^{\prime}$ oriented in equatorial coordinates and centred on the object position.

Table 1. VVV ZYJHK $K_{\mathrm{S}}$ magnitudes for V1309 Sco. The ZY and $J H K_{\mathrm{S}}$ data are quasi-simultaneous. Epochs for the $J H K_{\mathrm{s}}$ observations are marked in Fig. 2.

\begin{tabular}{lcccc}
\hline Filter & $\begin{array}{c}\lambda_{c} \\
{[\mu \mathrm{m}]}\end{array}$ & $\begin{array}{c}\text { PSF-mag } \\
{[\mathrm{mag}]}\end{array}$ & $\begin{array}{c}\text { Epoch } \\
{[\mathrm{JD}]}\end{array}$ & $\begin{array}{c}\text { Date } \\
{[\text { dd.mm.yyy }]}\end{array}$ \\
\hline$J$ & 1.254 & $13.849 \pm 0.007$ & 2455437 & 29.08 .2010 \\
$H$ & 1.646 & $12.973 \pm 0.008$ & 2455437 & 29.08 .2010 \\
$K_{\mathrm{S}}$ & 2.149 & $12.449 \pm 0.011$ & 2455437 & 29.08 .2010 \\
& & & & \\
$Z$ & 0.878 & $17.266 \pm 0.050$ & 2457282 & 15.09 .2015 \\
$Y$ & 1.021 & $16.629 \pm 0.032$ & 2457282 & 15.09 .2015 \\
$J$ & 1.254 & $15.080 \pm 0.025$ & 2457255 & 21.08 .2015 \\
$K_{\mathrm{S}}$ & 2.149 & $14.659 \pm 0.044$ & 2457255 & 21.08 .2015 \\
\hline
\end{tabular}

was obtained for both colour and variability data following the procedures described by Contreras Ramos et al. (2017) and Alonso-García et al. (2018). While the near-IR magnitudes of V1309 Sco are listed on Table 1, the PSF VVV $K_{\mathrm{S}}$-band light curve with 163 epochs is presented in Figure 2, along with SMARTS $K$-band data (Walter et al. 2012; McCollum et al. 2014).

From the previous light curve from SMARTS, it is interesting to note the presence of large near-IR variations at the late times, that might suggest a possible brightening of the target after $\sim 2013$ ( JD 2456000). However, this behaviour is not confirmed at all with our data-set, which shows a much tighter and smoother light-curve.

While the K-band in SMARTS is in the Johnson-Glass JHK system (Bessell \& Brett 1988), the $K_{\mathrm{S}}$ ( $K$ "short") band in VVV is in the VISTA photometric system (e.g., Sutherland et al. 2015; González-Fernández et al. 2018) ${ }^{2}$. The different band-passes of the two filters used for the observations, $K$ versus $K_{\mathrm{S}}$ band, where the effective wavelengths $\left(\lambda_{\text {eff }}\right)$ are $\sim 2.19$ and $2.149 \mu \mathrm{m}$ and the filter widths $(\Delta \lambda)$ are $\sim 0.39$ and $0.309 \mu \mathrm{m}$, respectively, cannot be the rea-

2 Transmission curves for the VISTA filters compared with other near-IR systems are presented in Fig. 3 of González-Fernández et al. (2018) 


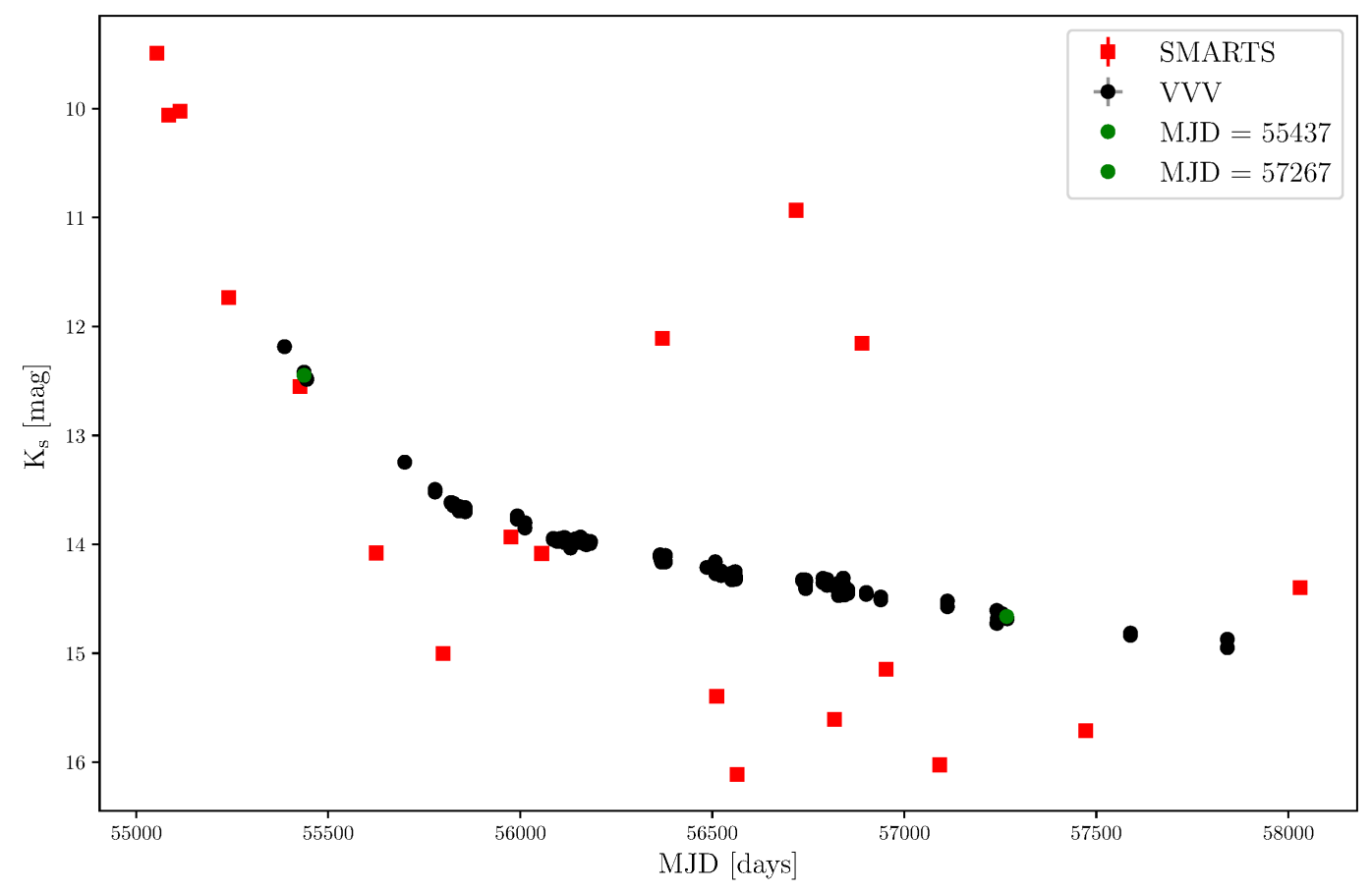

Figure 2. VVV $K_{\mathrm{S}}$-band light curve of V1309 Sco (black dots). There is a total of 163 epochs spanning from July 082010 to March 28 2017. SMARTS $K$-band observations are also shown in red dots. The $K$ and $K_{\mathrm{S}}$ passbands are close enough to allow the data-points be plotted in the same frame. Those green dots represents the $K_{\mathrm{S}}$ PSF magnitude in the years of 2010 and 2015 as demonstrated on Table 1 .

son for this difference, because very small photometric differences are expected in the mean $\left(\Delta_{K} \leq 0.03 \mathrm{mag}\right)$. Due to the presence of a slightly brighter star near V1309 Sco, we suggest that it was possibly blended with this source in some of the SMARTS observations, which would interfere at the shape of the aperture photometry light curve of the SMARTS project, as presented in Figure 2. We used in this study PSF photometry, considerably more accurate than the aperture photometry, therefore, the VVV data-set collected at the 4-meter VISTA Telescope should describe better its late behaviour.

\section{DATA ANALYSIS}

The VVV $K_{\mathrm{S}}$-band light curve (see Fig. 2) shows a smooth late behaviour for the V1309 Sco stellar merger remnant, with a small scatter in the $K_{\mathrm{S}}$-band, and also a slow decline in magnitude is presently levelling off, which does not match the behaviour from McCollum et al. (2014), as expected for a merger event.

When comparing our VVV $K_{\mathrm{S}}$-filter light curve with the OGLE I-band light curve presented by Tylenda et al. (2011) and later observations, we also observe that the source is steadily getting bluer. The colour in the year 2010 showed that the source was very red, with $\left(I-K_{\mathrm{S}}\right)=3.54 \mathrm{mag}$ and $\left(J-K_{\mathrm{S}}\right)=1.40 \mathrm{mag}$, and changing to $\left(I-K_{\mathrm{S}}\right)=2.75 \mathrm{mag}$ and $\left(J-K_{\mathrm{S}}\right)=0.42 \mathrm{mag}$ in the year of $2015^{3}$. Assuming the reddening for this field as $E\left(I-K_{\mathrm{S}}\right)=1.48 \mathrm{mag}$ from Schlafly \& Finkbeiner $(2011)$ yield unreddened colours $\left(I-K_{\mathrm{S}}\right)_{0}=$ 2.1 and $0.3 \mathrm{mag}$ for the years of 2010 and 2015, respectively. Similarly, $E\left(J-K_{\mathrm{S}}\right)=0.50 \mathrm{mag}$ from Schlafly \& Finkbeiner (2011) yields to $\left(J-K_{\mathrm{S}}\right)_{0}=0.9$ and $-0.1 \mathrm{mag}$ for 2010 and 2015. Those values imply either that the remnant star is changing its effective temperature, getting hotter with time, or that the dust column density is decreasing fast, as one would expect for an expanding dust shell for example.

\subsection{Searching for periodic variations}

Pre-outburst optical data of V1309 Sco present a periodic signal of $P_{0} \sim 0.7$ days with $\Delta K_{0} \sim 0.15 \mathrm{mag}$ (Tylenda et al. 2011). In order to search for periodic variations in our post-outburst data we applied a polynomial regression to our light curve to remove the long term variation (top panel of Figure 3). Thereafter, the residual light curve was calculated subtracting the original light curve by the polynomial, following $K_{\mathrm{S}}-\mathrm{FIT}$.

\footnotetext{
3 I-band magnitudes during year 2015 from SMARTS (http://www.astro.sunysb.edu/fwalter/SMARTS/NovaAtlas/ v1309sco/v1309sco.html)
} 

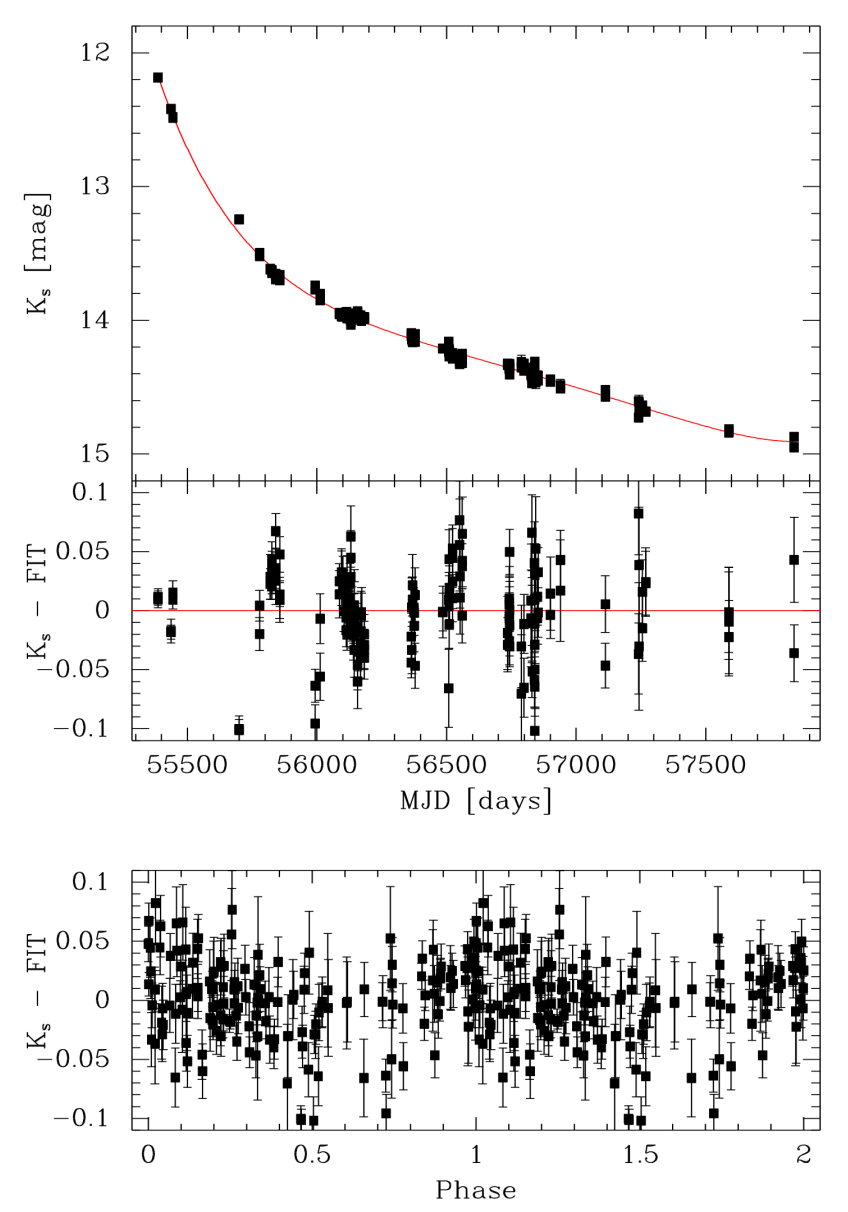

Figure 3. Top panel: post-outburst VVV $K_{\mathrm{S}}$-band light-curve (black dots) and the polynomial curve used to remove the long term variation (red curve). Central panel: the residual light curve calculated by subtracting the original light curve by the polynomial. Bottom panel: phase-folded light curve for the residual for a period of $P=0.498$ days (corresponding to $P \sim 12: 35$ hours) and a modulation amplitude of $\Delta K_{\mathrm{s}}=0.030 \mathrm{mag}$ (see Section 3).

Our search for a periodic component on the V1309 Sco post-outburst data was carried out using the Generalised Lomb-Scargle (GLS) method, also known as float mean periodogram (Zechmeister \& Kürster 2009), which provides a straightforward solution based on a Fourier-like power spectrum in order to detect and fit a sine-like periodic component at an unevenly-sampled data-set. Given a frequency grid spanning from two to fifty hours, the resultant period calculated with the method was $P=0.498194 \pm 0.000014$ days, corresponding to $P \sim 12: 35$ hours with a modulation amplitude of $\Delta K_{\mathrm{S}}=0.030 \pm 0.003 \mathrm{mag}$, notably smaller than $P_{0} \sim 0.7$ days and $\Delta K_{0} \sim 0.15 \mathrm{mag}$ presented by Tylenda et al. (2011). Even with a simple false alarm test estimating a good significance of this signal, the resultant period along with the relatively small amplitude must be seen with caution and can be interpreted as no longer the existence of a binary system post-2008 outburst. The phased light-curve of V1309 Sco for the period of $P=0.49$ days is presented on the bottom panel of Figure 3 .

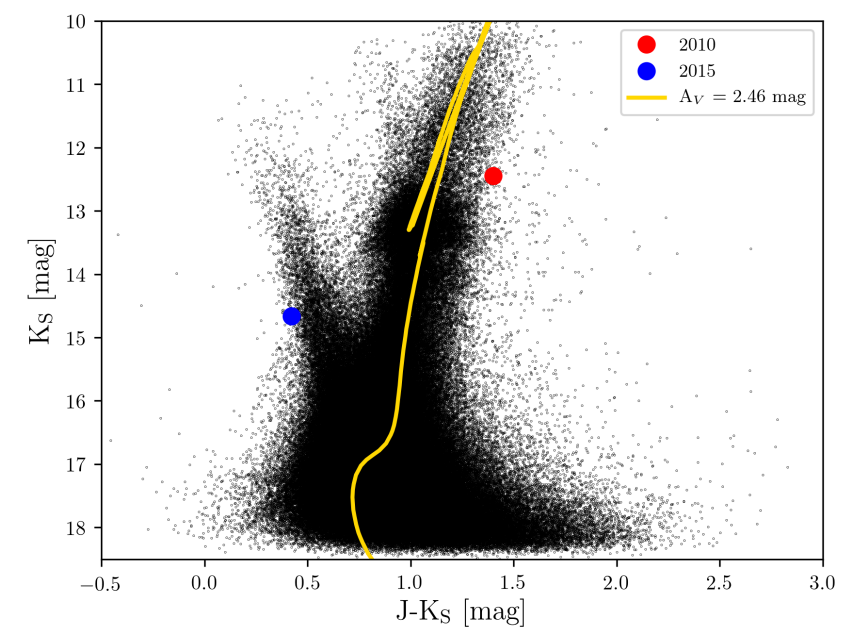

Figure 4. The PSF VVV J-KS vs. $K_{\mathrm{S}}$ colour-magnitude diagram (CMD) for 357,436 stars on tile b291 located within 15 arcmin radius of V1309 Sco. The red dot represents observations of the object in year 2010, while the blue dot represents observations of year 2015. An isochrone representing the Bulge population is also shown as a yellow curve (see Section 4).

\section{RESULTS AND DISCUSSION: THE ASYMPTOTIC BEHAVIOUR OF V1309 SCO}

The asymptotic near-IR magnitude and colours of V1309 Sco are $\mathrm{K}_{\mathrm{S}}=14.9 \pm 0.1,\left(\mathrm{~J}-\mathrm{K}_{\mathrm{S}}\right)_{0}=-0.10 \pm 0.05 \mathrm{mag}$ and $\left(\mathrm{I}-\mathrm{K}_{\mathrm{S}}\right)_{0}$ $=1.30 \pm 0.05 \mathrm{mag}$, respectively. While the colour are based on 2015 observations, the magnitude of $K_{S}=14.9$ mag refers to the latest data-point observed by the VVVX Survey, on March 28 2017. Regarding its asymptotic behaviour, our VVV K $\mathrm{K}_{\mathrm{S}}$-band observations during years 2016-2017 shows a very slow decline rate with slope $\Delta \mathrm{K}_{\mathrm{S}}=+0.12 \mathrm{mag} /$ year. Interestingly, there are some stellar sources in the VVV Survey database that show long-term variability, declining slowly and steadily with time, mimicking the late behaviour of the VVV Sco stellar merger.

Adopting the kinematic distance of $d=2.1 \mathrm{kpc}$ from Kamiński et al. (2018), and the field absorption of $\mathrm{A}_{K}=$ 0.37 mag from Schlafly \& Finkbeiner (2011), yields an absolute near-IR magnitude as $\mathrm{M}_{\mathrm{Ks}}=2.72 \mathrm{mag}$. The error in this magnitude is estimated to be $\sigma_{K_{\mathrm{S}}}=0.2 \mathrm{mag}$. We note that this is unlike a very luminous supergiant, and more consistent with a normal blue star. Figure 4 presents a colourmagnitude diagram from 15 arcmin radius region around the V1309 Sco position, made from PSF photometry of the VVV Survey data (Alonso-García et al. 2018). Overlaid to the CMD is an isochrone representing the Bulge population. It is based on PARSEC release v1.2S + COLIBRI $\mathrm{S}_{35}$ tracks ${ }^{4}$ (Pastorelli et al. 2019) for a stellar age of 10 Gy, solar metallicity, an extinction of $\mathrm{A}_{V}=2.46 \mathrm{mag}$ (see Section 2) and scaled for the distance of the Galactic centre (e.g. Rich 2001; Madrid 2018; Binney \& Merrifield 1998). It's important to note that V1309 Sco has changed its colour

${ }^{4}$ http://stev.oapd.inaf.it/cgi-bin/cmd 
at a relatively high rate, going from $\left(\mathrm{J}-\mathrm{K}_{\mathrm{S}}\right)=1.40 \mathrm{mag}$ in 2010 to $\left(\mathrm{J}-\mathrm{K}_{\mathrm{S}}\right)=0.42 \mathrm{mag}$ in 2015 . This significant colour change in a relatively short period of time shows that V1309 Sco was getting bluer and hotter with time, behaving as a blue straggler star (Sandage 1953).

There have been alternative explanations for the formation of the blue stragglers published in the past years, for example the mass transfer increasing in a binary system (e.g. McCrea 1964), the internal mixing of a single star due to fast rotation or the presence of a strong magnetic field (e.g. Wheeler 1979). The merger hypothesis should describe the nature of V1309 Sco. This states that blue stragglers spend a long lifetime as low-q binaries and result from the merger between two main sequence stars in dynamical interaction (e.g. Mateo et al. 1990; Lombardi et al. 2002). As the majority of blue stragglers are easily to identify in stellar clusters, both mass transfer and the collisional hypothesis seems to be equally possible (Mapelli et al. 2019). However, as pointed out by Leonard (1989), the collisional scenario fails to explain how those objects remain in the stellar cluster as the recoil velocity, in this case, should exceed the cluster escape velocity. Field stars like V1309 Sco are difficult to discover as blue stragglers because the main sequence turnoff (MSTO) point is not precisely defined in a field CMD. Therefore, V1309 Sco is an unique case where its path from a field star to the blue straggler regime has been followed.

Even the putative period of $P=0.49$ days in the postoutburst data of V1309 Sco could be related to a blue straggler. Especially in Mateo et al. (1990), some of the blue stragglers selected by their blue colour in the optical CMDs of the globular cluster NGC 5466 present periodic signals in the range $P=0.34-0.51$ days with amplitudes of $\Delta V=0.15-0.33$ mag, interpreted as the period of the binary system. While the periods are in good agreement with $P=0.49$ days for V1309 Sco, the amplitudes presented in Mateo et al. (1990) are much larger. However, we note that a different behaviour in the near-IR compared with the optical variability is expected for many classes of eclipsing and pulsating variables (e.g., Angeloni et al. 2014).

In conclusion, we find that reasonable equilibrium in this stellar merger is being reached rapidly. Only $\sim 9$ years after the outburst, V1309 Sco has settled into a nearly constant magnitude, resembling a normal blue star. The asymptotic blue colour of V1309 Sco as the resultant of a stellar merger suggests that the object is a "blue straggler" in the making, as theoretically predicted. With the current data we cannot conclusively establish the nature of the V1309 Sco remnant, thus it would be interesting to confirm if this behaviour persists for the following years, and continuous monitoring is desirable. V1309 Sco is the best studied stellar merger case to date and may become a laboratory to study the formation of blue straggler stars via stellar mergers.

\section{ACKNOWLEDGEMENTS}

We gratefully acknowledge the use of data from the ESO Public Survey program IDs 179.B-2002 and 198.B-2004 taken with the VISTA telescope, and data products from the Cambridge Astronomical Survey Unit (CASU). This publication makes use of VOSA, developed under the Spanish Virtual Observatory project supported from the Span- ish MINECO through grant AyA2017-84089. T.F. acknowledges support from PIBIC@UFSC and CNPq-Brazil. R.K.S. acknowledges support from $\mathrm{CNPq} /$ Brazil through projects 308968/2016-6 and 421687/2016-9. Support for the authors is provided by the BASAL CONICYT Center for Astrophysics and Associated Technologies (CATA) through grant AFB-170002, and the Ministry for the Economy, Development, and Tourism, Programa Iniciativa Científica Milenio through grant IC120009, awarded to the Millennium Institute of Astrophysics (MAS). D.M. acknowledges support from FONDECYT through project Regular \#1170121.

\section{REFERENCES}

Alonso-Garcia, J., Saito, R. K., Hempel, M., et al. 2018, A\&A, $619,4 \mathrm{~A}$

Angeloni, R., Contreras Ramos, R., Catelan, M., et al. 2014, A\&A, 567, A100

Banerjee, D. P. K., Hsiao, E. Y., Diamond, T., et al. 2018, ApJ, 867,99

Bessell, M. S., \& Brett, J. M. 1988, PASP, 100, 1134

Binney, J., \& Merrifield, M. 1998, Galactic astronomy / James Binney and Michael Merrifield. Princeton, NJ : Princeton University Press, 1998. (Princeton series in astrophysics) QB857.B522 1998

Cardelli, J. A., Clayton, G. C., \& Mathis, J. S. 1989, ApJ, 345, 245

Contreras Ramos, R., Zoccali, M., Rojas, F., et al. 2017, A\&A, 608, A140.

Gillessen S., Eisenhauer F., Trippe S., Alexander T., Genzel R., Martins F., Ott T., 2009, ApJ, 692, 1075

Gonzalez, O. A., Rejkuba, M., Zoccali, M., et al. 2012, A\&A, 543, A13

González-Fernández, C., Hodgkin, S. T., Irwin, M. J., et al. 2018, MNRAS, 474, 5459

Kamiński, T., Steffen, W., Tylenda, R., et al. 2018, A\&A, 617, A129

Kilic, M., Munn, J. A., Harris, H. C., et al. 2017, ApJ, 837, 162

Kochanek, C. S., Adams, S. M., \& Belczynski, K. 2014, MNRAS, 443, 1319

Leonard, P. J. T. 1989, AJ, 98, 217

Lombardi, J. C., Jr., Warren, J. S., Rasio, F. A., Sills, A., \& Warren, A. R. 2002, ApJ, 568, 939

Madrid, F. R. S. 2018 PhD Thesis, Ludwig-MaximilliansUniversit $\tilde{A} d$ 't

Mapelli, M., Giacobbo, N., Santoliquido, F., \& Artale, M. C. 2019, arXiv:1902.01419

Mason, E., Diaz, M., Williams, R. E., Preston, G., \& Bensby, T. 2010, A\&A, 516, A108

Mateo, M., Harris, H. C., Nemec, J., \& Olszewski, E. W. 1990, AJ, 100, 469

McCollum, B., Laine, S., Väisänen, P., et al. 2014, AJ, 147, 11

McCrea, W. H. 1964, MNRAS, 128, 147

Minniti, D. 2018, in The Vatican Observatory, Castel Gandolfo: 80th Anniversary Celebration (ed. G. Gionti, S.J., \& J.-B. Kikwaya Eluo, S.J). Astrophysics and Space Science Proceedings, 51, 63

Minniti, D., Lucas, P. W., Emerson, J. P., et al. 2010, New Astron., 15,433

Minniti, D., Saito, R. K., Forster, F., et al. 2017, ApJ, 849, L23

Nakano, S., Nishiyama, K., Kabashima, F., et al. 2008, IAU Circ., 8972, 1

Nandez, J. L. A., Ivanova, N., \& Lombardi, J. C., Jr. 2014, ApJ, 786,39

Nicholls, C. P., Melis, C., Soszynski, I., et al. 2013, MNRAS, 431, L33 
Nishiyama, S., Tamura, M., Hatano, H., et al. 2009, ApJ, 696, 1407

Paczyński, B. 1971, ARA\&A, 9, 183

Pastorelli, G., et al. 2019, submitted to MNRAS

Pietrukowicz, P., Soszyński, I., Udalski, A., et al. 2017, Acta Astron., 67, 115

Rich R. M., 2001, ASPC..245, 216, ASPC..245

Saito, R. K., Hempel, M., Minniti, D., et al. 2012, A\&A, 537, A107

Sandage, A. R. 1953, AJ, 58, 61

Schlafly, E. F., \& Finkbeiner, D. P. 2011, ApJ, 737, 103

Schlegel, D. J., Finkbeiner, D. P., \& Davis, M. 1998, ApJ, 500, 525

Smith, L., et al. 2018, in preparation

Sutherland, W., Emerson, J., Dalton, G., et al. 2015, A\&A, 575, A25

Tylenda, R., Hajduk, M., Kamiński, T., et al. 2011, A\&A, 528, A114

Tylenda, R., \& Kaminski, T. 2016, A\&A, 592, A134

Udalski, A., Szymanski, M. K., Soszynski, I., \& Poleski, R. 2008, Acta Astron., 58, 69

Walter, F. M., Battisti, A., Towers, S. E., Bond, H. E., \& Stringfellow, G. S. 2012, PASP, 124, 1057

Wheeler, J. C. 1979, ApJ, 234, 569

Zechmeister, M., \& Kürster, M. 2009, A\&A, 496, 577

Zhu, L.-Y., Zhao, E.-G., \& Zhou, X. 2016, Research in Astronomy and Astrophysics, 16, 68

This paper has been typeset from a $\mathrm{T}_{\mathrm{E}} \mathrm{X} / \mathrm{LAT}_{\mathrm{E}} \mathrm{X}$ file prepared by the author. 